\title{
Singular Value Decomposition
}

\author{
1 J. H. Caltenco, ${ }^{1}$ J. López-Bonilla, ${ }^{2}$ B. E. Carvajal-Gámez, ${ }^{3}$ P. Lam-Estrada, \\ 1 ESIME-Zacatenco, Instituto Politécnico Nacional (IPN), Edif. 5, CP 07738, México DF; \\ jlopezb@ipn.mx \\ 2 SEPI-ESCOM, IPN, Av. Bátiz S/N, Col. Nueva Industrial Vallejo, CP 07738, México DF, \\ ${ }^{3}$ ESFM-IPN, Depto. de Matemáticas, Edif. 9, Col. Lindavista, CP 07738, México DF
}

Keywords: Pseudoinverse of a matrix, SVD, Compatibility of linear systems

\begin{abstract}
We study the SVD of an arbitrary matrix $A_{n x m}$, especially its subspaces of activation, which leads in natural manner to pseudoinverse of Moore-Bjenhammar-Penrose. Besides, we analyze the compatibility of linear systems and the uniqueness of the corresponding solution, and our approach gives the Lanczos classification for these systems.
\end{abstract}

\section{Introduction}

For any real matrix $\mathrm{A}_{n x m}$, Lanczos [1] constructs the matrix:

$$
S_{(n+m) x(n+m)}=\left(\begin{array}{cc}
0 & A \\
A^{T} & 0
\end{array}\right),
$$

and he studies the eigenvalue problem:

$$
S \vec{\omega}=\lambda \vec{\omega},
$$

where the proper values are real because $\mathrm{S}$ is a real symmetric matrix. Besides:

$$
\operatorname{rank} A \equiv p=\text { Number of positive eigenvalues of } S \text {, }
$$

such that $1 \leq p \leq \min (n, m)$. Then the singular values or canonical multipliers, thus called by Picard [2] and Sylvester [3], respectively, follow the scheme:

$$
\lambda_{1}, \lambda_{2}, \ldots, \lambda_{p},-\lambda_{1},-\lambda_{2}, \ldots,-\lambda_{p}, 0,0, \ldots, 0,
$$

that is, $\lambda=0$ has the multiplicity $n+m-2 p$. Only in the case $p=n=m$ can occur the absence of the null eigenvalue.

The proper vectors of S, named ,essential axes ${ }^{\text {ee }}$ by Lanczos, can be written in the form:

$$
\vec{\omega}_{(n+m) x 1}=\left(\begin{array}{l}
\vec{u} \\
\vec{v}
\end{array}\right)_{m}^{n}
$$

then (1) and (2) imply the Modified Eigenvalue Problem:

$$
A_{n x m} \vec{v}_{m x 1}=\lambda \vec{u}_{n x 1}, \quad A_{m x n}^{T} \vec{u}_{n x 1}=\lambda \vec{v}_{m x 1},
$$

Thus

$$
A^{T} A \vec{v}=\lambda^{2} \vec{v}, \quad A A^{T} \vec{u}=\lambda^{2} \vec{u},
$$

with special interest in the associated vectors with the positive eigenvalues because they permit to introduce the matrices: 


$$
U_{n x p}=\left(\vec{u}_{1}, \vec{u}_{2}, \ldots, \vec{u}_{p}\right), \quad V_{m x p}=\left(\vec{v}_{1}, \vec{v}_{2}, \ldots, \vec{v}_{p}\right)
$$

verifying $U^{T} U=V^{T} V=I_{p x p}$ because:

$$
\vec{u}_{j} \cdot \vec{u}_{k}=\vec{v}_{j} \cdot \vec{v}_{k}=\delta_{j k}
$$

therefore $\vec{\omega}_{j} \cdot \vec{\omega}_{k}=2 \delta_{j k}, j, k=1,2, \ldots, p$. Thus, the SVD (Singular Value Decomposition) express [1, 4-6] that $A$ is the product of three matrices:

$$
A_{n x m}=U_{n x p} \Lambda_{p x p} V_{p x m}^{T}, \quad \Lambda=\operatorname{Diag}\left(\lambda_{1}, \lambda_{2}, \ldots, \lambda_{p}\right) .
$$

This relation tells that in the construction of A we do not need information about the null proper value; the information from $\lambda=0$ is important to study the existence and uniqueness of the solutions for a linear system associated to A. This approach of Lanczos is similar [7] to Schmidt [8] and Jordan [9, 10] methods; we can consider that Jordan, Sylvester [3] and Beltrami [11] are the founders of the SVD [12], and there is abundant literature [13-25] on this matrix factorization and its applications.

In Sec. 2, we realize an analysis of the proper vectors $\vec{\omega}_{j}, j=1, \ldots, n+m$, associated to the eigenvalues (4), which leads to the subspaces of activation of A with the pseudoinverse of Moore [26]-Bjerhammar [27]-Penrose [28]. In Sec. 3, we study the compatibility of linear systems, with special emphasis in the important participation of the null singular value and its corresponding eigenvectors.

\section{Subspaces of activation and natural inverse matrix}

From (6), the proper vectors associated with the positive eigenvalues verify:

$$
A \vec{v}_{j}=\lambda_{j} \vec{u}_{j}, \quad A^{T} \vec{u}_{j}=\lambda_{j} \vec{v}_{j}, \quad j=1, \ldots, p
$$

then

$$
A\left(-\vec{v}_{j}\right)=\left(-\lambda_{j}\right) \vec{u}_{j}, \quad A^{T} \vec{u}_{j}=\left(-\lambda_{j}\right)\left(-\vec{v}_{j}\right)
$$

that is

$$
S\left(\begin{array}{c}
\vec{u}_{k} \\
\vec{v}_{k}
\end{array}\right)=\lambda_{k}\left(\begin{array}{c}
\vec{u}_{k} \\
\vec{v}_{k}
\end{array}\right) \quad \text { implies } \quad S\left(\begin{array}{c}
\vec{u}_{k} \\
-\vec{v}_{k}
\end{array}\right)=\left(-\lambda_{k}\right)\left(\begin{array}{c}
\vec{u}_{k} \\
-\vec{v}_{k}
\end{array}\right) .
$$

Therefore, the eigenvectors $\left(\begin{array}{l}\vec{u}_{j} \\ \vec{v}_{j}\end{array}\right)$ and $\left(\begin{array}{c}\vec{u}_{j} \\ \vec{v}_{j}\end{array}\right)$ correspond to the proper values $\lambda_{1}, \ldots, \lambda_{p}$ and $-\lambda_{1}, \ldots,-\lambda_{p}$, respectively. Thus we must have $n+m-2 p$ eigenvectors connected to $\lambda=0$, which we denote by $\vec{\omega}_{r}^{(0)}$, and from (6):

$$
\begin{aligned}
\vec{\omega}_{j}^{(0)} & =\left(\begin{array}{c}
\vec{u}_{j}^{(0)} \\
0 \\
\vdots \\
0
\end{array}\right){ }_{m}^{n}, \quad A^{T} \vec{u}_{j}^{(0)}=\overrightarrow{0}, \quad j=1, \ldots, n-p, \\
\vec{\omega}_{(n-p)+k}^{(0)} & =\left(\begin{array}{c}
0 \\
\vdots \\
0 \\
\vec{v}_{k}^{(0)}
\end{array}\right)_{m}^{n}, \quad A \vec{v}_{k}^{(0)}=\overrightarrow{0}, \quad k=1, \ldots, m-p .
\end{aligned}
$$


The conditions (14) and (15) can be multiplied by $A$ and $A^{T}$, then $\vec{u}_{j}^{(0)}$ and $\vec{v}_{k}^{(0)}$ are eigenvectors of the Gram matrices $A A^{T}$ and $A^{T} A$ :

$$
\left(A A^{T}\right)_{n x n} \vec{u}_{j}^{(0)}=\overrightarrow{0}, \quad\left(A^{T} A\right)_{m x m} \vec{v}_{k}^{(0)}=\overrightarrow{0},
$$

but by (7) these matrices have $p$ proper vectors for $\lambda_{1}, \ldots, \lambda_{p}$, therefore only there are $n-p$ and $m-p$ vectors $\vec{u}_{j}^{(0)}$ and $\vec{v}_{k}^{(0)}$ that can be selected with orthonormality:

$$
\vec{u}_{j}^{(0)} \cdot \vec{u}_{r}^{(0)}=\delta_{j r}, \quad \vec{v}_{k}^{(0)} \cdot \vec{v}_{q}^{(0)}=\delta_{k q},
$$

that is, $\vec{\omega}_{j}^{(0)} \cdot \vec{\omega}_{k}^{(0)}=\delta_{j k}$, then $\left\{\vec{u}_{j}^{(0)}\right\}$ and $\left\{\vec{v}_{k}^{(0)}\right\}$ are bases for the Kernel $A^{T}$ and Kernel $A$, respectively.

If into (14) we employ (10) [SVD of $A$ ] results $V \Lambda U^{T} \vec{u}_{j}^{(0)}=\overrightarrow{0}$, whose multiplication by the left with $\Lambda^{-1} V^{T}$ [remembering that $\left.U^{T} U=V^{T} V=I\right]$, gives the compatibility condition:

$$
U^{T} \vec{u}_{j}^{(0)}=\overrightarrow{0} \quad \Rightarrow \quad \vec{u}_{r} \cdot \vec{u}_{j}^{(0)}=0, \quad r=1, \ldots, p ; j=1, \ldots, n-p,
$$

Equivalently

$$
\operatorname{Col} U \perp \vec{u}_{k}^{(0)}, \quad k=1, \ldots, n-p .
$$

Similarly, if we use SVD into (15) and we multiply by $\Lambda^{-1} U^{T}$ :

$$
\begin{gathered}
V^{T} \vec{v}_{k}^{(0)}=\overrightarrow{0}, \quad \vec{v}_{r} \cdot \vec{v}_{k}^{(0)}=0, \quad r=1, \ldots, p ; \quad k=1, \ldots m-p \\
\therefore \quad \text { Col } V \perp \vec{v}_{j}^{(0)}, \quad j=1, \ldots, m-p .
\end{gathered}
$$

It is convenient to make two remarks:

a).- From $A=U \Lambda V^{T}$ is evident that the matrices $U, \Lambda$ and $V$ permit to construct $A$, but is useful to know more about the structure of $A$ and its transpose:

$$
A=\left(\vec{a}_{1} \ldots \vec{a}_{m}\right), \quad A^{T}=\left(\vec{c}_{1} \ldots \vec{c}_{n}\right),
$$

where $\left(\vec{a}_{j}\right)_{n \times 1}$ and $\left(\vec{c}_{k}\right)_{m x 1}$ are the corresponding columns. Then from (10) we obtain the expressions:

$$
\vec{a}_{j}=\lambda_{1} v_{1}^{(j)} \vec{u}_{1}+\cdots+\lambda_{p} v_{p}^{(j)} \vec{u}_{p}, j=1, \ldots m, \quad \vec{c}_{k}=\lambda_{1} u_{1}^{(k)} \vec{v}_{1}+\cdots+\lambda_{p} u_{p}^{(k)} \vec{v}_{p}, \quad k=1, \ldots, n
$$

with the notation:

$$
v_{r}^{(j)}=j \text { th }- \text { component of } \vec{v}_{r}
$$

and similar for $u_{r}^{(k)}$; we observe that $\vec{c}_{k}^{T}$ are the rows of $A$. 
and similar for $u_{r}^{(k)}$; we observe that $\vec{c}_{k}^{T}$ are the rows of $A$.

From (23) are immediate the equalities of subspaces:

$$
\operatorname{Col} A=\operatorname{Col} U, \quad \text { Row } A=\operatorname{Col} V,
$$

but $\operatorname{dim} \operatorname{Col} U=\operatorname{dim} \operatorname{Col} V=p$, then:

$$
\text { rank } A=\operatorname{dim} \operatorname{Col} A=\operatorname{dim} \text { Row } A=p
$$

in according with (3).

).- We have the rank-nullity theorem [29-31]:

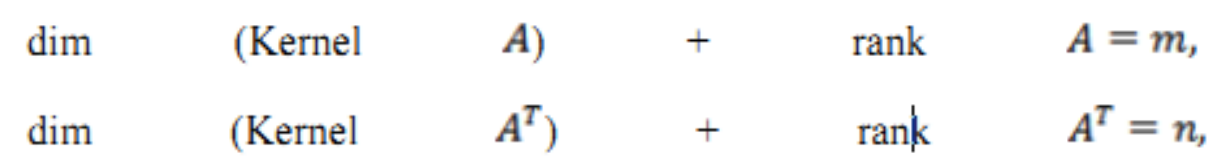

but $\operatorname{rank} A^{T}=\operatorname{rank} A=p$, then $\operatorname{dim}\left(\right.$ Kernel $\left.A^{T}\right)=n-p \quad$ in harmony with the $(n-p)$ vectors $\vec{u}_{j}^{(0)}$ verifying (14).

If $A_{n x m}$ acts on an arbitrary vector $\vec{x} \varepsilon E_{m}$ produces a vector $\vec{y} \varepsilon E_{n}$, with the decompositions:

$$
\begin{array}{cc}
\vec{x}=\vec{x}^{(0)}+\vec{x}_{\mathrm{CV}}, & \vec{y}=\vec{y}^{(0)}+\vec{y}_{\mathrm{CU}}, \\
\vec{x}=\vec{x}^{(0)}+\vec{x}_{\mathrm{CV}}, \quad \vec{y}=\vec{y}^{(0)}+\vec{y}_{\mathrm{CU}}, \\
\vec{x}^{(0)} \varepsilon \operatorname{Kernel} A, \quad \vec{x}_{\mathrm{CV}} \varepsilon \operatorname{Col} V, \quad A \vec{x}^{(0)}=\overrightarrow{0}, \quad \vec{x}^{(0)} \cdot \vec{x}_{\mathrm{CV}}=0,
\end{array}
$$

where

$$
\vec{y}^{(0)} \varepsilon \text { Kernel } A^{T}, \quad \vec{y}_{\mathrm{CU}} \varepsilon \operatorname{Col} U, \quad A^{T} \vec{y}^{(0)}=\overrightarrow{0}, \quad \vec{y}^{(0)} \cdot \vec{y}_{\mathrm{CU}}=0,
$$

Therefore, $A \vec{x}=A \vec{x}_{\mathrm{CV}}=\vec{y}$ and in the construction of $\vec{y}$ we lost the information about $\vec{x}^{(0)}$, then it is not possible to recover $\vec{x}$ from $\vec{y}$, that is, it is utopian to search for an 'inverse matrix' acting on $\vec{y}$ to give $\vec{x}$. However, when $\vec{x}^{(0)}=\overrightarrow{0}$ and $\vec{y}^{(0)}=\overrightarrow{0}$ we can introduce a 'natural inverse matrix', thus named it by Lanczos, which coincides with the pseudoinverse of Moore [16]-Bjerhammar [27]Penrose [28]:

"Any matrix $A_{n x m}$, restricted to its subspaces of activation, always can be inverted".

In fact, if $\vec{x} \varepsilon \mathrm{Col} V$ is an arbitrary vector, $\vec{x}=q_{1} \vec{v}_{1}+\cdots+q_{p} \vec{v}_{p}$, then from (6):

$$
A \vec{x}=\lambda_{1} q_{1} \vec{u}_{1}+\cdots+\lambda_{p} q_{p} \vec{u}_{p}=\vec{y} \varepsilon \operatorname{Col} U
$$

and now we search the inverse natural $A_{N \text { mxn }}^{-1}$ such that:

$$
A_{N}^{-1} \vec{y}=\vec{x} \text {, }
$$


or more general:

$$
A_{N}^{-1} A \vec{x}=\vec{x}, \quad \forall \vec{x} \varepsilon \operatorname{Col} V, \quad A A_{N}^{-1} \vec{y}=\vec{y}, \quad \forall \vec{y} \varepsilon \operatorname{Col} U .
$$

If the decomposition (10) is applied to (32) we deduce the natural inverse matrix:

$$
A_{N \text { mxn }}^{-1}=V_{m x p} \Lambda_{p x p}^{-1} U_{p x n}^{T}
$$

satisfying (33) and (34). With (35) is easy to prove the properties [24, 32]:

$$
A A_{N}^{-1} A=A, \quad A_{N}^{-1} A A_{N}^{-1}=A_{N}^{-1}, \quad\left(A A_{N}^{-1}\right)^{T}=A A_{N}^{-1}, \quad\left(A_{N}^{-1} A\right)^{T}=A_{N}^{-1} A,
$$

which characterize the pseudoinverse of Moore-Bjerhammar-Penrose, that is, the inverse matrix $[32,33]$ of these authors coincides with the natural inverse (35) deduced by Lanczos [1, 4-6].

which characterize the pseudoinverse of Moore-Bjerhammar-Penrose, that is, the inverse matrix [32, 33] of these authors coincides with the natural inverse (35) deduced by Lanczos [1, 4-6].

In the SVD only participate the positive proper values of $S$, without the explicit presence of the vectors $\vec{u}_{j}^{(0)}$ and $\vec{v}_{k}^{(0)}$ associated with the null eigenvalue, then it is natural to investigate the role performed by the information related with $\lambda=0$. In Sec. 3 , we study linear systems where $A$ is the corresponding matrix of coefficients, and we exhibit that the $\vec{u}_{j}^{(0)} \mid$ permit to analyze the compatibility of such systems; besides, when they are compatibles then with the $\vec{v}_{k}^{(0)}$ we search if the solution is unique. In other words, the null eigenvalue does not participates when we consider to $A$ as an algebraic operator and we construct its factorization (10), but $\lambda=0$ is important if $A$ acts as the matrix of coefficients of a linear system.

\section{Compatibility of linear systems}

A linear system of $n$ equations with $m$ unknowns can be written in the matrix form:

$$
A_{n \times m} \vec{x}_{m \times 1}=\vec{b}_{n \times 1}
$$

where (10) implies that $U \Lambda V^{T} \vec{x}=\vec{b}$ whose multiplication by $\vec{u}_{j}^{(0) T}$ gives the compatibility conditions:

$$
\vec{u}_{j}^{(0)} \cdot \vec{b}=0, \quad j=1, \ldots, n-p
$$

due to (19). Then the system (37) is compatible if $\vec{b}$ is orthogonal to all independent solutions of the adjoint system $A^{T} \vec{u}=\overrightarrow{0}$, therefore:

$$
" A \vec{x}=\vec{b} \quad \text { has solution if } \vec{b} \varepsilon \operatorname{Col} U ",
$$

which is the traditional formulation [6] of the compatibility condition for a linear system given. From (25) and (39) is clear that $A$ and the augmented matrix $(A \vec{b})$ have the same column space:

$$
\operatorname{Col} A=\operatorname{Col}(A \vec{b})=\operatorname{Col} U
$$

thus at the books [32] we find the result:

$$
" A \vec{x}=\vec{b} \quad \text { is compatible if } \quad \operatorname{rank} A=\operatorname{rank}(A \vec{b}) " .
$$


If $\vec{b} \varepsilon \operatorname{Col} U$, then from (11):

$$
\vec{b}=b^{(1)} \vec{u}_{1}+\cdots+b^{(p)} \vec{u}_{p}=A \vec{Q}, \quad \vec{Q}=\frac{b^{(1)}}{\lambda_{1}} \vec{v}_{1}+\cdots+\frac{b^{(p)}}{\lambda_{p}} \vec{v}_{p}
$$

and (37) leads to:

$$
A(\vec{x}-\vec{Q})=\overrightarrow{0}
$$

The set of solutions of (43) is the Kernel $A$ with dimension $(m-p)$ due to (27), therefore (43) has the unique solution $\vec{x}-\vec{Q}=\overrightarrow{0}$ when $p=m$, that is, when rank $A$ coincides with the number of unknowns we have not vectors $\vec{v}_{k}^{(0)} \neq \overrightarrow{0}$ verifying $A \vec{v}_{k}^{(0)}=\overrightarrow{0}$. Then:

"The compatible system $A \vec{x}=\vec{b}$ has unique solution only when $p=m^{\prime}$, $x_{r}=Q^{(r)}=\frac{b^{(1)}}{\lambda_{1}} v_{1}^{(r)}+\cdots+\frac{b^{(p)}}{\lambda_{p}} v_{p}^{(r)}=\vec{b} \cdot \vec{t}_{r}, \quad r=1, \ldots, m$

where

$$
\vec{t}_{r}=\frac{v_{1}^{(r)}}{\lambda_{1}} \vec{u}_{1}+\cdots+\frac{v_{p}^{(r)}}{\lambda_{p}} \vec{u}_{p} \quad \varepsilon \operatorname{Col} U
$$

thus the value of each unknown is the projection of $\bar{b}$ onto each vector (46). In consequence, $\vec{b} \varepsilon$ Col $U$ guarantees the solution of (37), and it is unique only if $p=m$.

Besides, from (42) we see that the solution $\vec{x}=\vec{Q}$ implies that $\vec{x} \varepsilon \operatorname{Col} V$, then we have the system $A \vec{x}=\vec{b}$ where $\vec{x}$ and $\vec{b}$ are totally embedded into Col $V$ and Col $U$, respectively, that is, $\vec{x}$ and $\vec{b}$ are into the subspaces of activation of $A$, thus from (32) and (33) there is the natural inverse $A_{N}^{-1}$ such that:

$$
\vec{x}=A_{N}^{-1} \vec{b}=V_{m x m} \Lambda_{m x m}^{-1} U_{m x n}^{T} \vec{b}=V \Lambda^{-1}\left(\begin{array}{c}
b^{(1)} \\
1 \\
b^{(m)}
\end{array}\right)=V\left(\begin{array}{c}
\frac{b^{(1)}}{\lambda_{1}} \\
1 \\
\frac{b^{(m)}}{\lambda_{m}}
\end{array}\right)=\left(\begin{array}{c}
\frac{b^{(1)}}{\lambda_{1}} v_{1}^{(1)}+\ldots+\frac{b^{(m)}}{\lambda_{m}} v_{m}^{(1)} \\
\frac{b^{(1)}}{\lambda_{1}} v_{1}^{(m)}+\ldots+\frac{b^{(m)}}{\lambda_{m}} v_{m}^{(m)}
\end{array}\right), p=m,
$$

in according with (45). The vectors (46) are important because their inner products with $\vec{b}$ give the solution of (37) via (45), and they also are remarkable because permit to construct the natural inverse:

$$
A_{N \text { mxn }}^{-1}=\left(\vec{t}_{1} \vec{t}_{2} \ldots \vec{t}_{m}\right)^{T}, \quad p=m .
$$

Lanczos [6] considers three situations:

i). $n<m$ : The linear system is under-determined because it has more unknowns than equations, and from $1 \leq p \leq \min (n, m)$ is impossible the case $p=m$, therefore, if (37) is compatible then its solution cannot be unique.

ii). $n=m$ : The system is even-determined with unique solution when $p=m$, that is, if $\operatorname{det} A \neq 0$. In this case also $p=n$, we have not vectors $\vec{u}_{j}^{(0)} \neq \overrightarrow{0}$, thus $\vec{b} \varepsilon \operatorname{Col} U$ and automatically the system is compatible.

iii). $n>m$ : The linear system is over-determined, and by $1 \leq p \leq \min (n, m)$ can occur the case $p=m$ for unique solution if the system is compatible.

Thus it is immediate the classification of linear systems introduced by Lanczos [6]:

Free and complete:

$p=n=m$, unique solution,

Restricted and complete: $\quad p=m<n, \quad$ over-determined, unique solution, 
Free and incomplete: $\quad p=n<m$, under-determined, non-unique solution,

Restricted and incomplete: $p<n$ and $p<m$, solution without uniqueness,

with the meanings:

Free: $\quad$ The conditions (30) are satisfied trivially.

Restricted: It is necessary to verify that $\vec{b} \varepsilon \operatorname{Col} U$.

When $p \neq m$, the homogeneous system $A \vec{v}=\overrightarrow{0}$ has the non-trivial solutions $\vec{v}_{k}^{(0)}$, then from (27) we conclude that the general solution of (37) is:

$$
\vec{x}=\vec{Q}+c_{1} \vec{v}_{1}^{(0)}+\cdots+c_{m-p} \vec{v}_{m-p}^{(0)}
$$

where the ${ }^{c_{k}}$ are arbitrary constants.

\section{Conclusions}

With the SVD we can find the subspaces of activation of , and it leads to natural inverse [6, 2628] of any matrix, known it in the literature as the Moore-Penrose pseudoinverse. Besides, the SVD gives a better understanding of the compatibility of linear systems. On the other hand, Lanczos [6] showed that the Singular Value Decomposition provides a universal platform to study linear differential and integral operators for arbitrary boundary conditions.

\section{References:}

[1]. C. Lanczos, Linear systems in self-adjoint form, Am. Math. Monthly 65, No. 9 (1958) 665-679 [2]. É. Picard, Sur un theorem general relative aux integrals de premier espéce et sur quelques problémes de physique mathématique, Rend. Circ. Mat. Palermo 25 (1910) 79-97

[3]. J. J. Sylvester, Sur la réduction biorthogonale d'une forme linéo-linéaire á sa forme cannonique, Compt. Rend. Acad. Sci. Paris 108 (1889) 651-653

[4]. C. Lanczos, Extended boundary value problems, Proc. Int. Congr. Math. Edinburgh-1958, Cambridge University Press (1960) 154-181

[5]. C. Lanczos, Boundary value problems and orthogonal expansions, SIAM J. Appl. Math. 14, No. 4 (1966) 831-863

[6]. C. Lanczos, Linear differential operators, Dover, New York (1997)

[7]. F. Smithies, Linear differential operators, The Mathematical Gazette 47 (1963) 265-266

[8]. E. Schmidt, Zur theorie der linearen und nichtlinearen integralgleichungen, Teil 1, Mathematische Annalen Bd. 63 (1907) 433-476 and 64 (1907) 161-174

[9]. C. Jordan, Mémoire sur les forms bilinéaires, J. de Mathématiques Pures et Appliquées, Deuxieme Série 19 (1874) 35-54

[10]. C. Jordan, Sur la réduction des formes bilinéaires, Compt. Rend. Acad. Sci. Paris 78 (1874) 614-617

[11]. E. Beltrami, Sulle funzioni bilineari, Giornale di Mathematische 11 (1873) 98-106

[12]. G. W. Stewart, On the early history of the SVD, SIAM Review 35 (1993) 551-566

[13]. J. J. Sylvester, On the reduction of a bilinear quantic of the nth order to the form of a sum of $n$ products, Messenger of Mathematics 19 (1889) 42-46

[14]. L. Autonne, Sur les matrices hypohermitiennes et sur les matrices unitaries, Compt. Rend. Acad. Sci. Paris 156 (1913) 858-860

[15]. E. T. Browne, The characteristic roots of a matrix, Bull. Amer. Math. Soc. 36, No. 10 (1930) 705-710

[16]. C. Eckart, G. Young, A principal axis transformation for non-hermitian matrices, Bull. Amer. Math. Soc. 45, No. 2 (1939) 118-121

[17]. H. Schwerdtfeger, Direct proof of Lanczos decomposition theorem, Am. Math. Monthly 67, No. 9 (1960) 855-860 
[18]. I. J. Good, Some applications of the singular decomposition of a matrix, Technometrics 11, No. 4 (1969) 823-831

[19]. C. Long, Visualization of matrix singular value decomposition, Mathematics Magazine 56, No. 3 (1983) 161-167

[20]. S. J. Blank, N. Krikorian, D. Spring, A geometrically inspired proof of the singular value decomposition, Am. Math. Monthly 96, No. 3 (1989) 238-239

[21]. D. Kalman, A singularly valuable decomposition: The SVD of a matrix, The College Mathematics Journal 27 (1996) 2-23

[22]. G. Golub, Aspects of scientific computing, Johann Bernoulli Lecture, Univ. of Groningen, 8th April 1996

[23]. V. Gaftoi, J. López-Bonilla, G. Ovando, Singular value decomposition and Lanczos potential, in "Current topics in quantum field theory research", Ed. O. Kovras, Nova Science Pub., New York (2007)

[24]. H. Yanai, K. Takeuchi, Y. Takane, Projection matrices, generalized inverse matrices, and singular value decomposition, Springer, New York (2011) Chap. 3

[25]. I. Guerrero M., J. López-Bonilla, L. Rosales R., SVD applied to Dirac supermatrix, The SciTech, J. Sci.\& Tech. (India), Special Issue, Aug. 2012, 111-114

[26]. E. H. Moore, On the reciprocal of the general algebraic matrix, Bull. Am. Math. Soc. 26, No. 9 (1920)394-395

[27]. A. Bjerhammar, Application of calculus of matrices to method of least squares, with special references to geodetic calculations, Trans. Roy. Inst. Tech. Stockholm No. 49 (1951) 1-86

[28]. R. Penrose, A generalized inverse for matrices, Proc. Camb. Phil. Soc. 51 (1955) 406-413 